\title{
LES and FW-H Prediction of Aeroacoustic Noise for a SD 7037 Airfoil for Wind Turbine Applications
}

\author{
Alison Zilstra* and David A. Johnson ${ }^{\dagger}$ \\ University of Waterloo, Waterloo, Ontario, Canada, N2L 3G1
}

\begin{abstract}
The aeroacoustic noise generated by wind turbines poses issues with the implementation of this renewable energy technology. The use of a fully analytical model for predicting airfoil noise could serve as a crucial tool in the design phase of new turbines or noise reduction technologies. This work uses a combination of Large Eddy Simulation (LES) and the Ffowcs-Williams and Hawkings (FW-H) acoustic model to predict the noise generated by a 2D segment of the SD 7037(c) airfoil. The simulations are performed at a static angle of attack (AOA) and at a Reynolds number typical for small scale wind turbines of $R e=4.3 \times 10^{4}$. The flow and acoustic results are validated against experimental results conducted by the Wind Energy Group at the University of Waterloo. This model was able to accurately predict the flow field and acoustic results for the $0^{\circ} \mathbf{A O A}$, and determined the source of the $4.1 \mathrm{kHz}$ tone to be 2D vortex shedding from the trailing edge (TE) and the $3.4 \mathrm{kHz}$ tone to come from the transition from 2D to 3D boundary layer behaviour. The $1^{\circ}$ AOA simulation, while able to simulate the flow and broadband acoustic spectra, requires further investigation to simulate the complex boundary layer transition behaviours required to predict the $3.4 \mathrm{kHz}$ tone. Overall, this method proved to be an effective predictive tool for airfoil self-noise at static AOAs.
\end{abstract}

\section{Nomenclature}

$c_{o} \quad=$ speed of sound $[\mathrm{m} / \mathrm{s}]$

$C_{L} \quad=$ lift coefficient

$C_{p} \quad=$ pressure coefficient

$\delta \quad=$ Dirac delta function

$d t \quad=$ time step $[s]$

$f(\vec{x}, t)=$ Surface of interest for FW-H Analogy

$p_{i} j \quad=$ compressive stress tensor $[\mathrm{Pa}]$

$\rho \quad=$ density $\left[\mathrm{kg} / \mathrm{m}^{3}\right]$

$T_{i j} \quad=\quad$ Lighthill's Tensor

\section{Introduction}

$\mathrm{T}$ HIS paper summarizes the research conducted for the computational aeroacoustic (CAA) modelling of the SD 7037 airfoil for wind turbine applications. The selected airfoil is well suited for use on small horizontal axis wind turbines due to its lift and drag characteristics [1]. Since the public acceptance of wind turbines is negatively impacted by the aeroacoustic noise they produce, it is important to develop accurate prediction tools for aeroacoustic noise. Currently, semi-empirical acoustic models allow for acoustic prediction, but are unable to predict noise from new blade designs or new noise reducing technologies. In order to reduce the noise, fully analytical prediction tools must be developed to allow acoustic analysis during the design stage of a wind turbine. With the increasing computational power of computational fluid dynamics (CFD) software, it is possible to perform complex acoustic simulations of full-blade or full-turbine models to determine the aeroacoustic noise emitted by the specific turbine. Building confidence in these models requires validation of simpler, controlled cases before applying the prediction model to a full wind turbine. This paper examines the accuracy of aeroacoustic noise simulations of a 2D SD 7037(c) airfoil at static angles of attack (AOA) through the comparison with experimental results. The simulations are conducted as a two step process: first the

\footnotetext{
*PhD Candidate, Wind Energy Group, Mechanical and Mechatronics Engineering, amzilstr@uwaterloo.ca, Student Member

†Professor, Wind Energy Group, Mechanical and Mechatronics Engineering, David.johnson@uwaterloo.ca
} 
flow around the airfoil is simulated using Large Eddy Simulation (LES) to generate the pressure and velocity field, and second the pressure data on the airfoil surface is propagated to the receiver by the Ffowcs-Williams and Hawkings (FW-H) acoustic analogy to predict the far field sound. These simulations are conducted using ANSYS Fluent, and are validated against experimental data conducted at the same conditions [2].

\section{Background and Theory}

\section{A. Wind Turbine Noise}

Wind turbines produce broadband noise as the rotating blades interact with the incoming wind. The modern megawatt (MW) scale wind turbines largely experience "turbulent boundary layer - trailing edge" (TBL-TE) noise, which originates from the turbulent boundary layer of the blade interacting at the trailing edge of the airfoil [3]. This interaction causes pressure fluctuations that are persistent enough to create aeroacoustic noise. The majority of the noise is generated at the trailing edge of the blade, and in the third of the blade closest to the tip. This portion of the blade has the highest relative wind velocity and therefore is most likely be operating with high levels of turbulence. The aeroacoustic noise from the blade is only present on one side due to the directivity of the noise produced by the TBL-TE acoustic mechanism, and therefore it can only be heard when the blade is in the down-sweep of its rotation (moving from top to bottom) [3]. This pattern can be seen in Figure 1] Smaller, lower Reynolds number wind turbines produce similar acoustic behaviour, but are also capable of producing tonal noise from the "laminar boundary layer - vortex shedding" (LBL-VS) airfoil self-noise mechanism. The LBL-VS noise is generated from vortex shedding from the trailing edge (TE) of the airfoil, as well as more complex feedback loops responsible for boundary layer phenomena such as a laminar separation bubble.
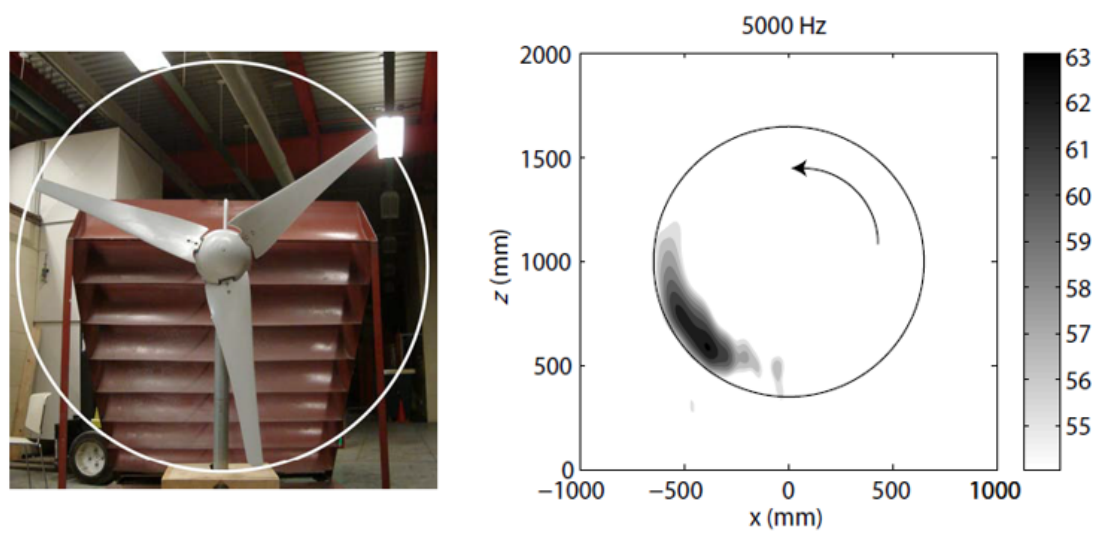

Fig. 1 Acoustic source map of a small wind turbine conducted by Bale using a MEMS microphone phased array [4]

In 2017, Tam published a study on airfoil self-noise for wind turbine applications which included acoustic measurements on a SD-7037 airfoil in a closed loop wind tunnel [5]. These measurements were taken at both static and oscillating AOAs at $R e=4.3 \times 10^{4}$ to understand the aeroacoustic noise under the dynamic conditions wind turbines operate in when installed. The experimental setup is shown in Figure 2. The static AOA measurements conducted by Tam serve as the validation for the simulated acoustic results.

Experiments for the flow and laminar separation bubble behaviour of the SD-7037 airfoil were conducted by Ghorbanishohrat in 2019 [6]. The work collected high quality Particle Image Velocimetry (PIV) data at the same Reynolds number as Tam and therefore will serve as validation for the simulated flow. The primary data used is static lift coefficients, surface pressure coefficients and averaged velocity field data.

\section{B. Computational Fluid Dynamics}

Predicting the aeroacoustic noise from an object requires an accurate simulation of the flow around the object, including the pressure and velocity both on the surface and near to the surface. The CFD models used for the numerical prediction are Reynolds-Averaged Navier-Stokes (RANS) and Large Eddy Simulation (LES). The RANS simulation 


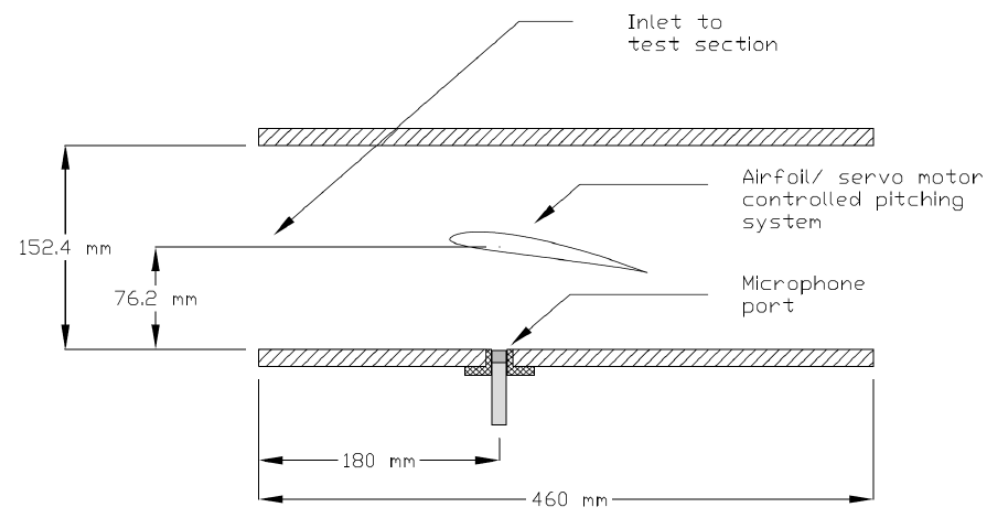

Fig. 2 SD-7037 test setup for Tam experiments [5]

is used to provide a more realistic starting point for the LES simulation which reduces the time for the simulation to become statistically stable [2]. The LES simulation is run simultaneously with the acoustic model to determine the transient behaviour of the flow field. Specific RANS and LES model settings are described in the Numerical Setup section.

\section{Computational Aeroacoustics}

The FW-H analogy is a rearrangement of mass and momentum conservation into an inhomogeneous wave equation that accounts for the presence of an impermeable surface in the flow. The resulting equation has three inhomogeneous terms: a quadrupole term which accounts for sound generated by fluctuating Reynolds stresses, a monopole (or thickness noise) term and a dipole (or loading noise) term. Together, the thickness and loading noise terms represent the sound generated by the body passing through the flow [7]. In Eq. 11, the quadrupole term contains Lighthill's Tensor, $T_{i j}$, the loading noise term contains the compressive stress tensor, $p_{i j}$, and the thickness noise term contains the fluid velocity, $u_{i}[8]$.

$$
\left(\frac{\partial^{2}}{\partial t^{2}}-c_{o}^{2} \frac{\partial^{2}}{\partial x_{i}^{2}}\right)\left(\rho^{\prime} H(f)\right)=\frac{\partial^{2}}{\partial x_{i} \partial x_{j}}\left(T_{i j} H(f)\right)-\frac{\partial}{\partial x_{i}}\left(p_{i j} \delta(f) \frac{\partial f}{\partial x_{j}}\right)+\frac{\partial}{\partial t}\left(\rho_{0} u_{i} \delta(f) \frac{\partial f}{\partial x_{i}}\right)
$$

The function, $f(\vec{x}, t)=0$, defines the surface of the body and therefore the quadrupole term applies outside of the defined surface, and the thickness and loading noise terms only apply on the surface of the body. In the case of airfoil self-noise, the quadrupole term is often neglected since the noise generation is dominated by the thickness and loading noise terms [9]. The solution of the FW-H acoustic analogy used for this model is Formulation 1A by Farassat [9], which places an impermeable surface on the blade and calculates the sound propagation using a retarded time frame. Overall, computing the aeroacoustic noise emitted from a turbine blade requires an understanding of the flow behaviour around the blade. The key parameters needed for acoustic prediction are the pressure and fluid velocity at the surface of the airfoil. This data must be simulated first using the LES simulation before the FW-H equations are applied to predict the aeroacoustic noise.

ANSYS Fluent has a built-in FW-H solver that has a similar solution to Formulation 1A [7]. The main difference is the solution uses a semi-permeable surface that can be offset from the airfoil to compute the quadrupole noise for the flow contained within the surface [2]. However, when placed coincident to the airfoil surface, the calculation simplifies to the Formulation 1A solution. The latter method was used for the prediction model.

\section{Numerical Setup}

\section{A. Geometry and Mesh}

The experimental geometry is a $25 \mathrm{~mm}$ airfoil with a span of $152.4 \mathrm{~mm}$, within a closed loop wind tunnel with a square test section of $152.4 \mathrm{~mm} \times 152.4 \mathrm{~mm}$. The geometry for the simulation is a C-Mesh with a boundary offsets of 20 times the chord length and a span 1/15 of the experimental span. To avoid discrepancies between the simulated and 
experimental Sound Pressure Levels (SPL), 15 microphones were added to the simulation as shown in Figure 3 to gather an acoustic signal that represents the entire span of the experimental airfoil.

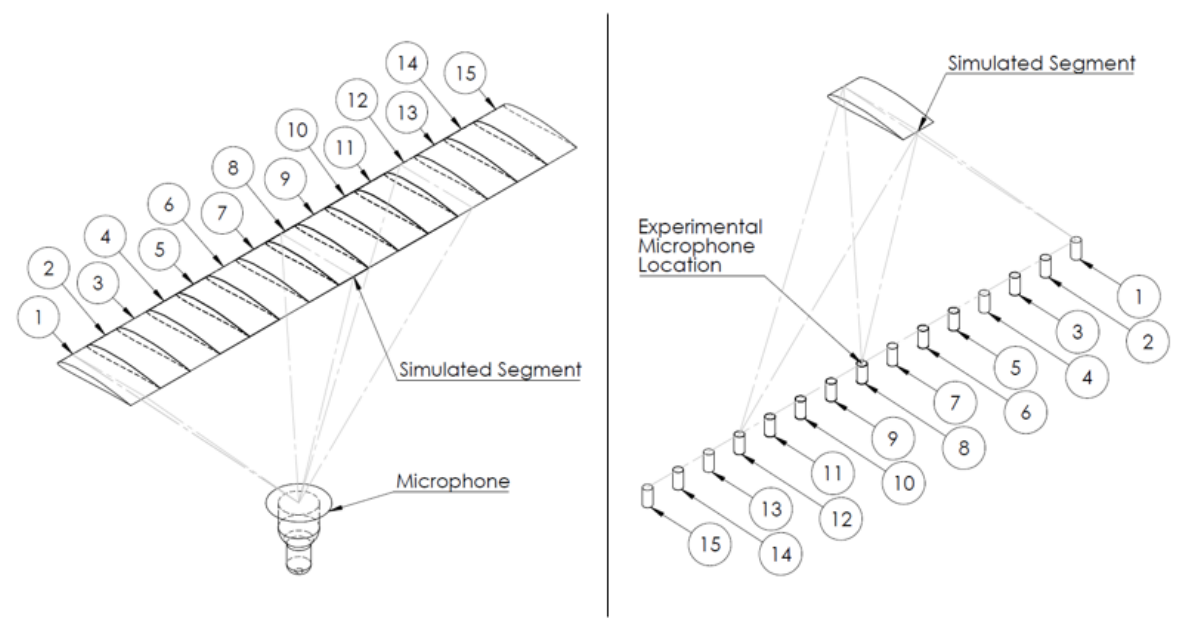

Fig. 3 SD-7037 Relative microphone locations for simulation setup

The mesh does not include the tunnel walls as the effects are assumed negligible on the flow behaviour around the airfoil. The mesh was broken down into two sections to accurately simulate: (a) the boundary layer and wake of the airfoil,and (b) the far field (Figure 4a). The boundary layer segment is set up such that $y^{+}<1,8$ to 10 elements are within the boundary layer and there is a smooth transition to the outer mesh [10]. The far field area allows for expansion of the mesh while maintaining realistic aspect ratios. The mesh has a total of 7 million cells and to ensure accurate simulation of the pressure fluctuations on the surface of the airfoil, there are 200 nodes along the chord of the airfoil (Figure $4 \mathrm{~b}$ ).

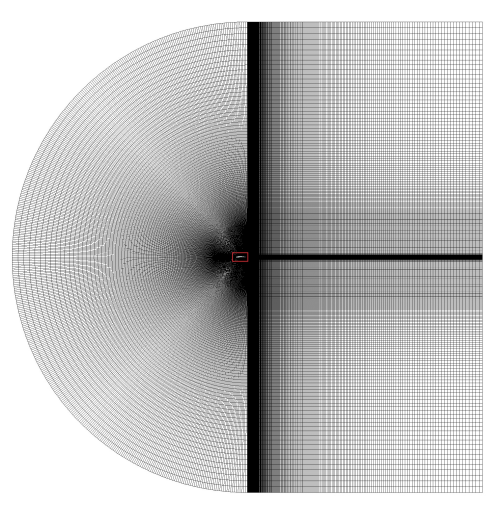

(a)

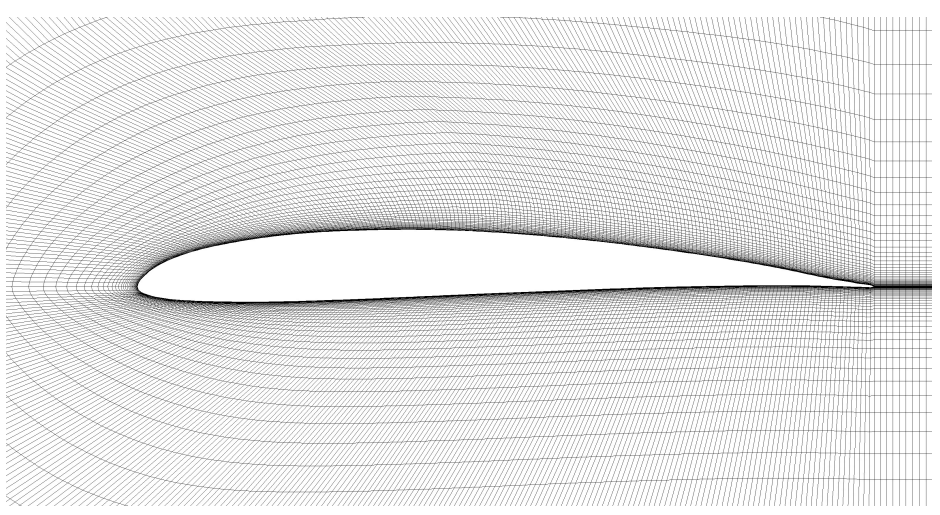

(b)

Fig. 4 C-Mesh for SD 7037, with box indicating airfoil close-up view

The inlet for the C-Mesh is specified as the front curved surface as well as the top and bottom horizontal surfaces. The velocity components are set for the given angle such that the velocity magnitude is $24.8 \mathrm{~m} / \mathrm{s}$ with a turbulent intensity of $1.23 \%$ and length scale of $0.0107 \mathrm{~m}$ to be consistent with the experiments conducted by Tam [5]. The back vertical boundary is set as a pressure-outlet. Both the inlet and outlet have the Non-Reflecting condition set for the acoustics model. The side surfaces are set as symmetry and the airfoil surface is set as a non-slip wall. 


\section{B. Model Parameters and Solver Setup}

The RANS initialization was run with the realizable $k-\epsilon$ model, with standard wall functions. The pressure-velocity coupling scheme used was SIMPLEC with Second Order Upwind spatial discretization. Convergence criteria was set at $1 \times 10^{-6}$ for all residuals. After convergence was achieved, the instantaneous flow field was generated using the built in Fluent command init-instantaneous-vel [2].

For the transient solution, Fluent's built in models for LES and FW-H were applied along with energy equation and ideal gas calculation of density to account for compressibility [2]. The LES subgrid-scale model was chosen as Dynamic-Stress Smagorinsky-Lilly. The acoustics model defined the source as the surface of the airfoil and the receiver locations as those shown in Figure 3 The acoustic data was exported at each timestep and calculated after the completion of the transient simulation. The time step was set at $1 \times 10^{-6}$ and convergence criteria was set at $5 \times 10^{-5}$ for all residuals. As with the RANS initialization, the SIMPLEC scheme was used, with Second Order discretization for pressure and Bounded Second Order Implicit for the transient formulation. The solution was run for two Mean Flow Residence Times (MFRT) with acoustic data omitted for the first MFRT to allow for a statistically steady solution.

The simulations are performed using the high performance computer cluster Graham, which is a part of the SHARCNET network and the greater Compute Canada network [11] [12]. The simulations were generally run using one complete node of the system, which contains 32 cores and required 3.25 weeks of execution time, or 1 month when including wait times for resources, to meet desired MFRT goal. The FW-H results, post-processing and graphics were performed in Fluent on a local computer with 8 cores and 32GB RAM.

\section{Results}

\section{A. Average Flow Data Comparison}

The results presented in this paper are for static AOAs of $0^{\circ}$ and $1^{\circ}$, and are compared against $1^{\circ}$ AOA averaged flow data from Ghorbanishohrat, as well as $0^{\circ}$ and $1^{\circ} \mathrm{AOA}$ acoustic data from Tam [6] [5]. First, the flow behaviour on the surface of the airfoil is analysed using pressure coefficient, $C_{p}$, and lift coefficient, $C_{L}$, in Figure 5 . The experimental $C_{p}$ results were processed from the PIV data by Ghorbanishohrat, and are shown for $1^{\circ}$ and $3^{\circ}$ [6]. The results for each of the simulated AOAs are very consistent for $0<x / c<0.9$, with differences occurring at the trailing edge of the airfoil. The most significant trend in this plot is the alignment of the $0^{\circ} \mathrm{AOA}$ simulated data with the $1^{\circ} \mathrm{AOA}$ experimental data, and that the $1^{\circ} \mathrm{AOA}$ simulated data is located between the $1^{\circ}$ and $3^{\circ} \mathrm{AOA}$ experimental data. This likely indicates that there are different $0^{\circ} \mathrm{AOA}$ locations for the simulations and the experiments, when considering the boundary layer behaviour of the airfoil. The exact cause of the difference is unknown but it is known that airfoils at low Reynolds number flow and low AOAs experience complex laminar to turbulent boundary layer transition behaviours that are very sensitive to small changes in the flow. Since this sensitivity is present for this setup, the source of the differences could be due to the effects of the tunnel walls on the experimental boundary layer behaviour or errors in the compressible LES simulation of the flow, or both. With that being said, the magnitude and trend in the averaged $C_{p}$ data are in good agreement. This agreement is also found in the $C_{L}$ data presented in Figure $5 \mathrm{~b}$

The mean velocity field is a useful comparison tool for transient simulations since it shows the overall trend for the boundary layer and wake fluctuations. For this comparison, the velocity is broken down into X and Y components and then divided by the inlet velocity magnitude of $24.8 \mathrm{~m} / \mathrm{s}$ to create a non-dimensional result. Figure 6 shows the $\mathrm{X}$-direction mean velocity fields and Figure 7 shows the Y-direction mean velocity fields for all four cases and compares them to experimental PIV data from Ghorbanishohrat at $R e=42,500$ [6]. Overall, the X-velocity contours are very similar with differences appearing along the pressure side of the airfoil (bottom of airfoil) and in the trailing edge region of the suction side (top of airfoil). For the pressure side, the velocity contours are very similar for each simulated AOA, but the $0^{\circ}$ result has overall a higher velocity than the $1^{\circ}$ result. When this is compared with the experimental results, both AOAs have a slightly higher velocity than the PIV results, but still contain the same pattern. The TE region differs when considering the dark blue region of negative velocity, where the $0^{\circ} \mathrm{AOA}$ has a this region extending a little farther into the wake than the $1^{\circ} \mathrm{AOA}$. All simulated data shows this region with a stronger negative velocity than the experimental data, but the overall size and shape of the region is the same. 


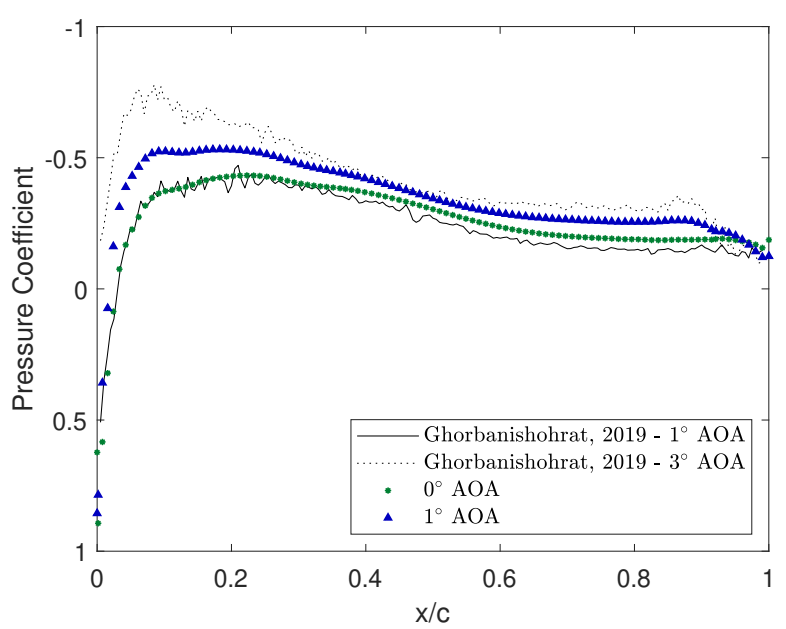

(a)

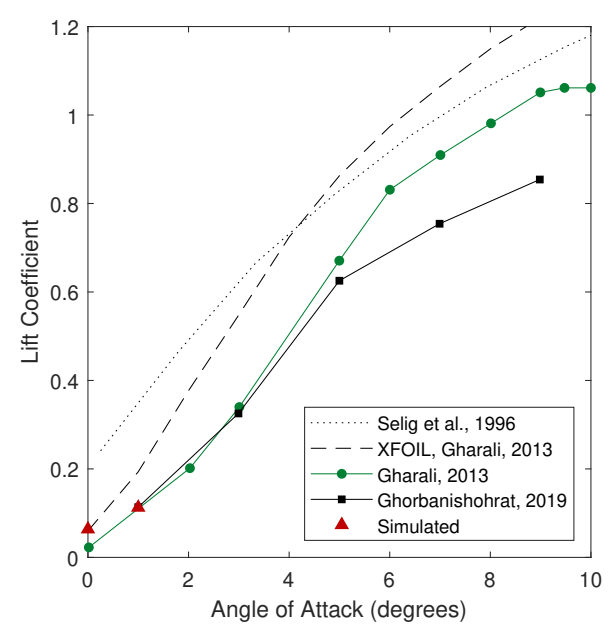

(b)

Fig. 5 (a) $0^{\circ}$ and $1^{\circ}$ AOA mean pressure coefficient versus $x / c$ compared with experimental data by Ghorbanishohrat [6]. (b) Mean lift coefficient values compared experimental data by Selig et al. [13] for $\operatorname{Re}=6 \times 10^{4}$, XFOIL $\left(R e=6 \times 10^{4}\right)$ and PIV results by Gharali [14] and PIV results by Ghorbanishohrat [6]

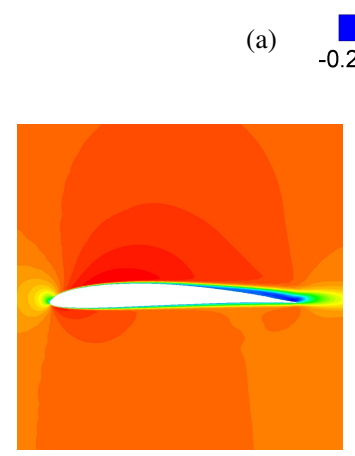

(b)

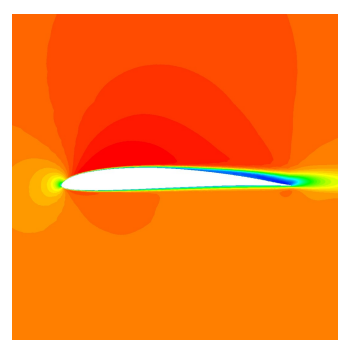

(c)

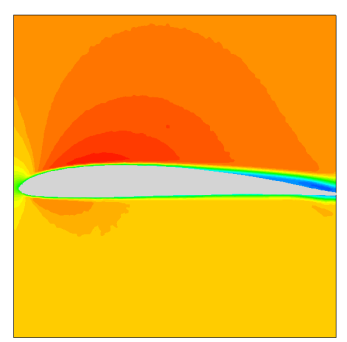

(d)

Fig. 6 Mean X velocity for (b) $0^{\circ}$ and (c) $1^{\circ}$ AOA, compared with (d) PIV results by Ghorbanishohrat [6]. Velocities are non-dimensionalized with the free stream velocity.

The $\mathrm{Y}$ velocity data also is in good agreement, though there are larger differences between the cases than in the $\mathrm{X}$ velocity data. The experimental Y velocity field shown in Figure 7d shows a consistent pattern of alternating low and high velocity regions in the wake, indicating that the vortices shed in the experimental case remain consistent with time. For the simulated results these vortices are less consistent with time and are therefore not visible in the averaged data. The $0^{\circ} \mathrm{AOA}$ data is very similar in both magnitude and pattern except for the vortex pattern in the wake and the $1^{\circ}$ data is also similar with a small increase in velocity magnitude. As with the $\mathrm{X}$-velocity, there are general differences between the simulated and experimental results, with an overall increase in Y-velocity occurring for the simulations and also a decrease in the strength of the positive velocity regions at the TE and on the suction side of the airfoil near the TE. These differences in the trailing edge and wake behaviour could be seen in the $C_{P}$ plot in Figure 5 a 
(a)

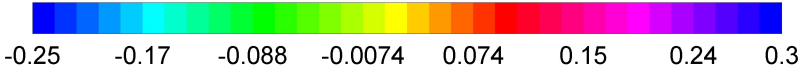

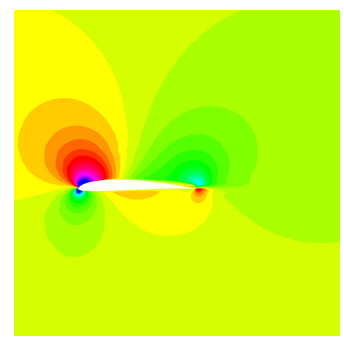

(b)

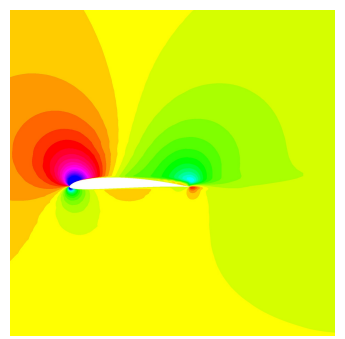

(c)

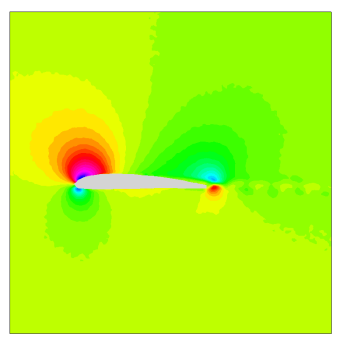

(d)

Fig. 7 Mean Y velocity for (b) $0^{\circ}$ and (c) $1^{\circ}$ AOA, compared with (d) PIV results by Ghorbanishohrat [6]. Velocities are non-dimensionalized with the free stream velocity magnitude.

\section{B. Boundary Layer and Wake Behaviour}

The above flow comparisons were made using averaged data, which is useful for validating the overall behaviour of the flow simulation. For acoustic prediction, it is also important to accurately simulate the transient behaviour in the flow since the changing pressure data on the surface of the airfoil is the key input into the FW-H acoustic model. Figure $8 \mathrm{a}$ and $8 \mathrm{~b}$ show the $C_{L}$ time history for the final $0.02 s$ of the $0^{\circ} \mathrm{AOA}$ and $1^{\circ} \mathrm{AOA}$ simulations, respectively. Both lift coefficient histories show low frequency fluctuation about the mean value at approximately $0.1 \mathrm{kHz}$, which is not a significant frequency for the airfoil self noise spectra of this airfoil, but it does follow changes in the flow behaviour. When looking at the $0^{\circ} \mathrm{AOA}$ result, there are consistent fluctuations that occur when the lift coefficient is below the mean value, and when above the mean value exhibits no regular pattern. This behaviour is also present in the $1^{\circ} \mathrm{AOA}$ data, but not to the same extent.

(a)

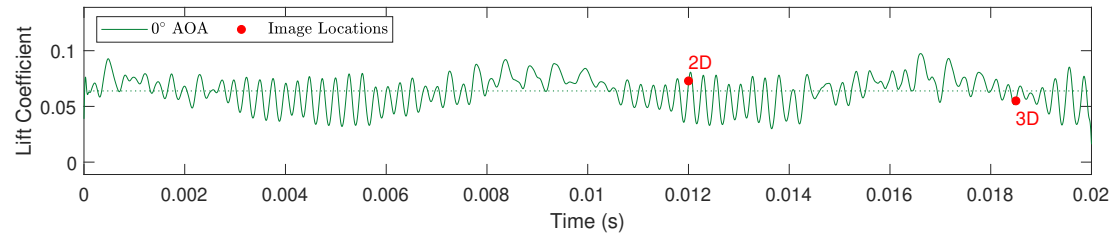

(b)

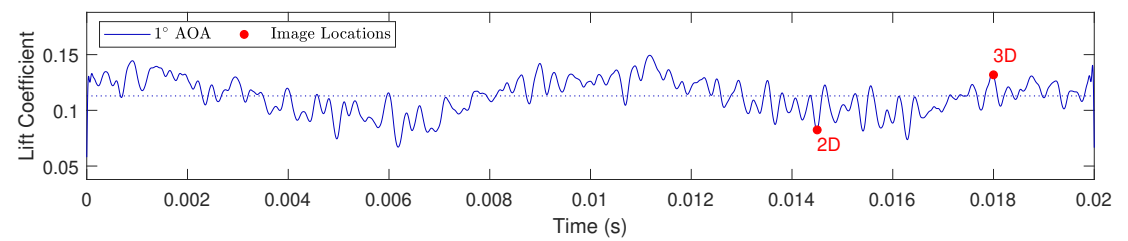

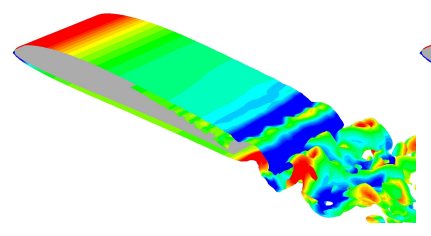

(c) $0^{\circ} \mathrm{AOA}-2 \mathrm{D}$

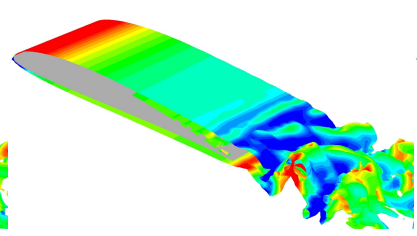

(d) $0^{\circ} \mathrm{AOA}-3 \mathrm{D}$

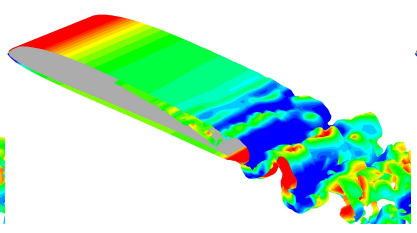

(e) $1^{\circ} \mathrm{AOA}-2 \mathrm{D}$

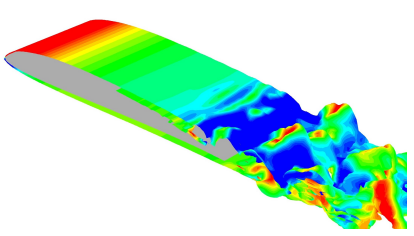

(f) $1^{\circ} \mathrm{AOA}-3 \mathrm{D}$

$(\mathrm{g})$

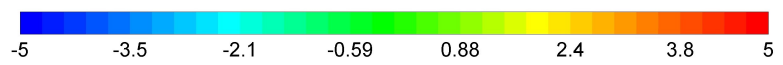

Fig. 8 Lift coefficient time history for the final $0.02 s$ of the (a) $0^{\circ}$ and (b) $1^{\circ}$ AOA simulations. (c)-(f) $0^{\circ}$ and $1^{\circ}$ AOA iso-surface of Vorticity Magnitude $=100001 / \mathrm{s}$ coloured with Y-Velocity from -5 to $+5 \mathrm{~m} / \mathrm{s}$

An examination of the flow behaviours responsible for these patterns is shown in Figures $8 \mathrm{c}$ to $8 \mathrm{f}$, which is a visual 
comparison of the vorticity in the boundary layer and the wake. The figures show an iso-surface generated in Fluent where the vorticity magnitude is $10,000[1 / s]$, that is coloured with a contour of Y velocity limited to the range of \pm 5 $\mathrm{m} / \mathrm{s}$. These are instantaneous snapshots of the simulated flow behaviour, and the time steps they are taken from are labelled on the $C_{L}$ time history for the corresponding AOA (Figure $8 \mathrm{a}$ and $8 \mathrm{~b}$ ). For each AOA, the images are labelled as either $2 \mathrm{D}$ or $3 \mathrm{D}$, which refers to the dimensionality of the wake behaviour. The $2 \mathrm{D}$ wake behaviour for $0^{\circ} \mathrm{AOA}$, shown in Figure $8 \mathrm{c}$. shows an undulating pattern indicating that there are vortices being shed from the trailing edge of the airfoil. This image is taken from the region of the $C_{L}$ time history where there are regular fluctuations, and the wake behaviour was found to be consistent throughout the regularly fluctuating regions. It is most likely that the fluctuations seen in the lift time history is due to the vortices being continuously shed from the TE and altering the pressure distribution near the trailing edge of the airfoil. Another important note is that there is no spanwise variation in the wake which is why this wake behaviour is labelled as $2 \mathrm{D}$.

Figure $8 \mathrm{~d}$ shows the $0^{\circ} \mathrm{AOA} 3 \mathrm{D}$ wake behaviour that occurs in the regions of the $C_{L}$ time history above the mean value, where there are no regular fluctuations occurring. The wake is behaving differently across the span of the airfoil, so the vortices that are shed do not have a combined impact on the lift properties of the airfoil and therefore do not create a regular pattern in the lift coefficient time history. For the 3D wake, the beginning of the transition from 2D to 3D behaviour occurs further upstream from the trailing edge, and therefore the transition point must be shifting upstream and downstream during the changes in lift coefficient behaviour. This change from 2D to 3D behaviour is an indication of the complex behaviours that occur in the transition from a laminar to turbulent boundary layer. The difference between the laminar and turbulent boundary layer is important for the prediction of airfoil self-noise mechanisms, since a laminar boundary layer (LBL-VS) is capable of producing both tonal and broadband noise and a turbulent boundary layer (TBL-TE) can only generate broadband noise.

The $1^{\circ}$ AOA data also changes between having a 2D wake and a 3D wake, but unlike the $0^{\circ}$ AOA data, the majority of the time it has a 3D wake as opposed to a 2D wake. Since the AOA is a degree higher, it is expected that any patterns in the boundary layer behaviour shift from the trailing edge toward the leading edge. Figure $8 \mathrm{e}$ shows the 2D wake behaviour for $1^{\circ} \mathrm{AOA}$, which occurs when the lift coefficient is below the average value. When compared to $0^{\circ} \mathrm{AOA}$, the 2D wake is not as well defined and appears to be on the verge of transition to a 3D wake. This absence of a well defined 2D wake explains why the lift coefficient time history for $1^{\circ}$ AOA does not have a regularly fluctuating region like it does in $0^{\circ} \mathrm{AOA}$. The final image of the vorticity iso-surface is for the $3 \mathrm{D}$ wake for $1^{\circ} \mathrm{AOA}$ (Figure $8 \mathrm{f}$ ), which closely resembles the behaviour of the $3 \mathrm{D}$ results for $0^{\circ} \mathrm{AOA}$.

\section{Acoustic Signal Comparison}

The acoustic data was calculated by the FW-H model using the second MFRT of the LES simulation, which resulted in an acoustic pressure signal $0.0405 \mathrm{~s}$ long for each of the 15 receivers. The acoustic pressure signal for each receiver is a result of the application of the FW-H acoustic analogy to the pressure distribution on the surface of the airfoil. The signal from each microphone is identical, with slight phase shifts as a result of the extra distance travelled between the airfoil and the receiver. Figure 9 , shows the signal for receiver 8 which is aligned with the mid-plane of the airfoil span (i.e. directly below the airfoil). The signals have had a bandpass filter applied to clearly show the frequencies ranging between 1 and $10 \mathrm{kHz}$. These signals are shown for the final $0.02 \mathrm{~s}$ of the measured signal, which is also the same times used for the lift coefficient histories shown above.

The acoustic signal for both simulations show almost identical behaviour to its lift history. The $0^{\circ}$ AOA results, shown in Figure 9a, has the same sections of regular frequency and high amplitude separated by sections of lower amplitude oscillations of varying frequencies. From this, it can be shown that the lift coefficient time history is a good indicator of the acoustic signal behaviour. In Figure 8a, it was shown that the regular frequency fluctuations of the lift coefficient correspond to 2D behaviour in the wake, and since these fluctuations are present in the acoustic signal, it can be concluded that the 2D wake is responsible for the high amplitude fluctuations in the acoustic signal. There is no dominant frequency present in the acoustic signal for the $1^{\circ}$ AOA data, which follows similar behaviour to the lift coefficient. This is also true for the irregular regions found in the $0^{\circ} \mathrm{AOA}$ signal. The irregular frequencies can be tied back to the spanwise variation in the flow found in the 3D wake behaviour of the $1^{\circ}$ AOA data (Figure $8 \mathrm{~b}$ ).

The microphone measurements made by Tam are presented as a signal of voltage versus time for AOAs of $0^{\circ}, 1^{\circ}$, and $3^{\circ}$ in Figures 9c 9e [5]. As with the simulation results, a bandpass filter has been applied with the range of 1 and $10 \mathrm{kHz}$ to highlight the key frequencies in the signal. Since the microphone data is presented as voltage, a direct comparison of the signal amplitudes is not possible but a useful comparison of signal behaviour is possible. The experimental voltage signals show a very low amplitude and inconsistent frequency fluctuation at $0^{\circ}$ which then jumps to a high amplitude 
and consistent frequency at $1^{\circ}$. The $1^{\circ}$ experimental signal most closely resembles the acoustic pressure signal of the $2 \mathrm{D}$ regions of the $0^{\circ} \mathrm{AOA}$ signal, and the $0^{\circ}$ experimental signal does not appear to match the patterns predicted in the simulation. The experimental signal for $3^{\circ}$ shows the narrow range of AOA in which the tonal noise occurs, with Tam finding a gradual decrease in signal amplitude while maintaining a relatively consistent frequency from $1^{\circ}$ to $3^{\circ}$ AOA [5]. The frequency and amplitude patterns in the simulated $1^{\circ}$ results seem to match the closest with those in the $3^{\circ}$ experimental signal. Interestingly, the none of the recorded signals have the cyclical behaviour that is found in the simulated results, indicating that the flow behaviour is more consistent with time.

(a)

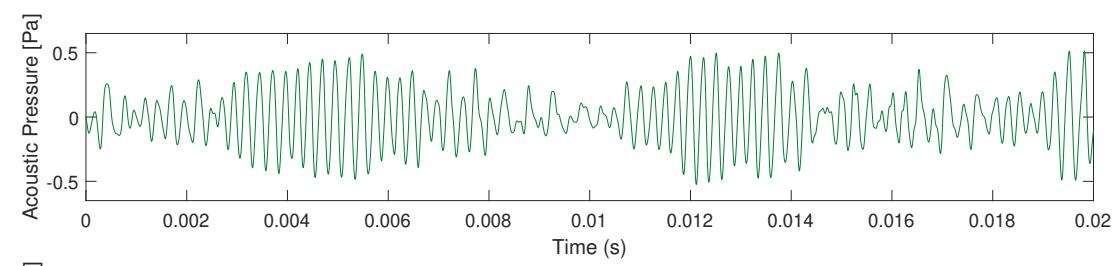

(b)

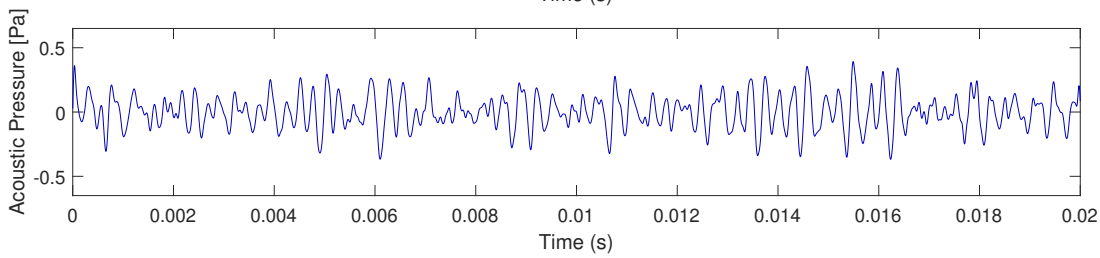

(c)

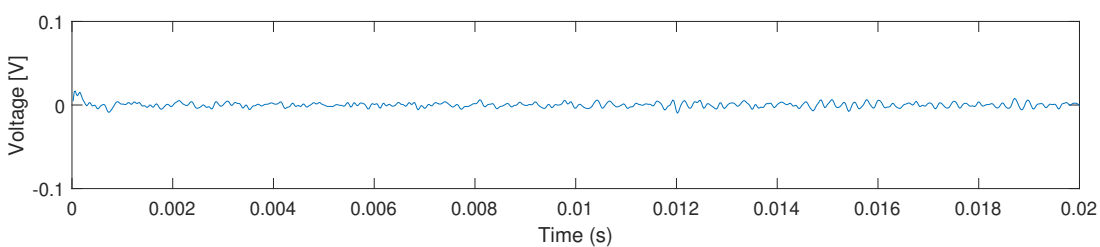

(d)
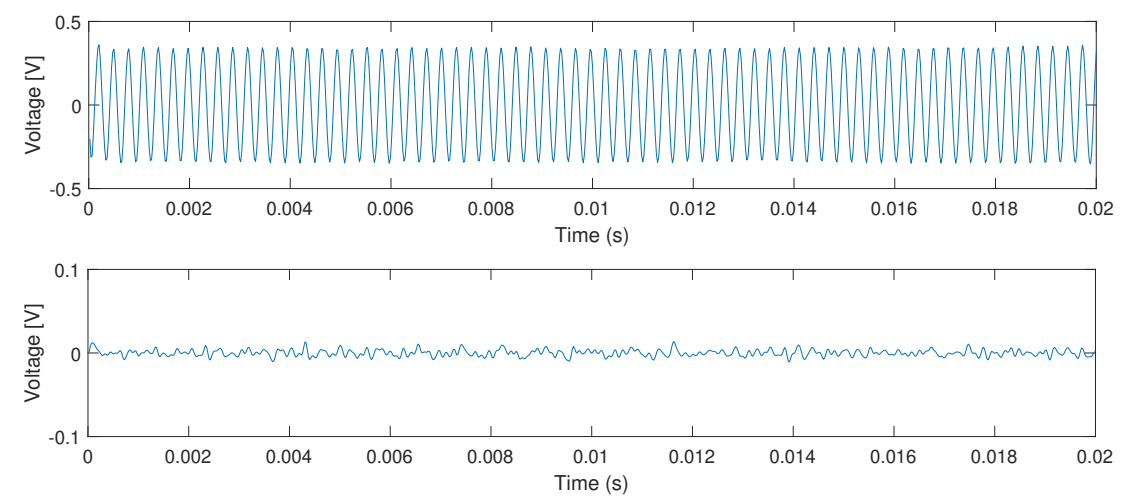

Fig. 9 (a) $0^{\circ}$ and (b) $1^{\circ}$ AOA acoustic pressure signal $(\mathrm{Pa})$ at the receiver for the final 20,000 time steps. And (c) $0^{\circ}$, (d) $1^{\circ}$, and (e) $3^{\circ}$ AOA voltage signal $(V)$ for $0.02 s$ measured by Tam [5]

\section{Acoustic Spectra Comparison}

The acoustic signals for all 15 receiver locations were processed using the FFT algorithm in Matlab with a Hamming window applied, and the acoustic pressure for each frequency band was then converted to SPL. For each frequency band, the SPL for each receiver was added together to get the total SPL for the airfoil span equivalent to the experimental span. The results for each case are presented in Figure 10, and are compared against the acoustic spectra measured by Tam for the same AOA [5]. The spectra results for Tam's experiments were produced with an ensemble average of the FFT results, which were performed on signal segments $1 s$ in length. The simulated signals were processed for a length of $0.0405 s$, and resulted in wider frequency bins in the narrowband spectra and prevents a direct comparison of SPL. To counteract this, the experimental bins were added together to result in the same bin widths as the simulated results. These plots highlight the two frequencies in the experiments where sharp peaks occurred, indicating tonal noise. These were measured at $3.4 \mathrm{kHz}$ and $4.1 \mathrm{kHz}$, and are indicated on the plots with vertical dotted lines. 


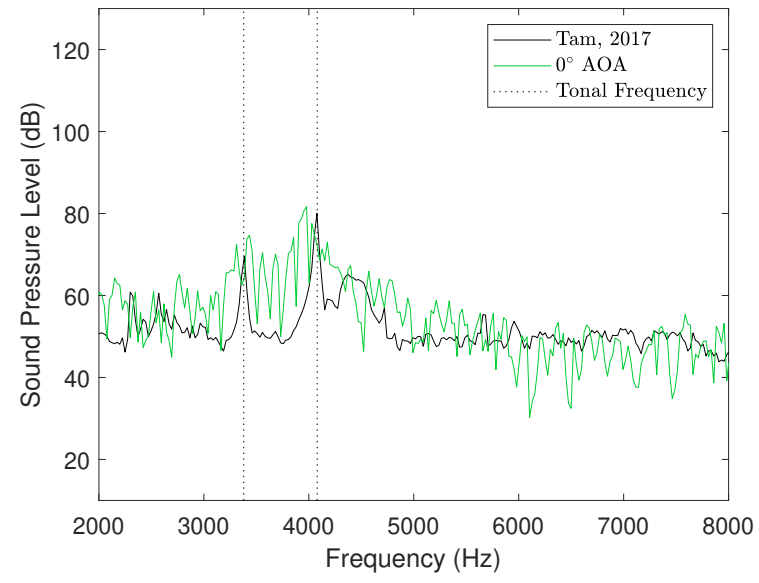

(a)

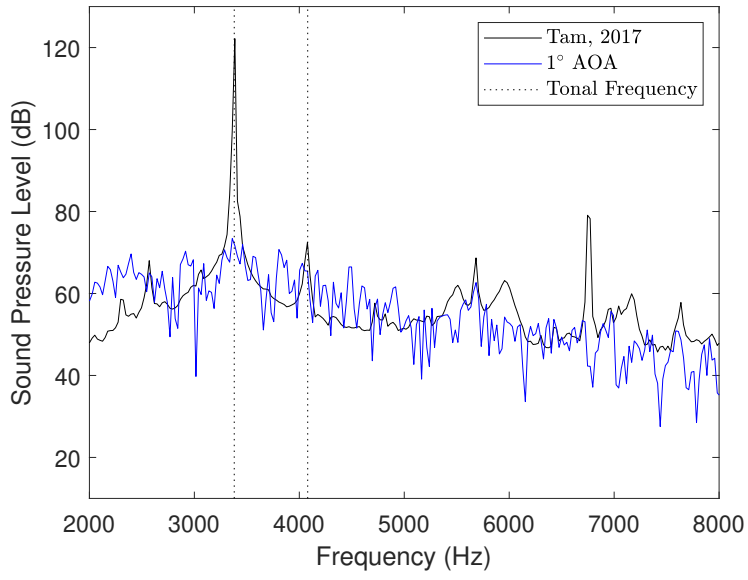

(b)

Fig. 10 Narrowband acoustic spectra for (a) $0^{\circ}$ and (b) $1^{\circ}$ AOA. Results plotted against experimental data of the same angle from Tam [5]

The simulated $0^{\circ} \mathrm{AOA}$ results match the trend in the acoustic spectra with the simulated data having a coarser appearance with sharp peaks and troughs. This is a result of the short simulated signal time which led to an inability to average the FFT results to gain smoother peaks. A longer acoustic pressure signal would produce narrower frequency bin widths as well as allow for splitting of the signal into smaller segments that could be processed separately and averaged. With this coarser data, it is still possible to analyze the trend in the data around the locations of the experimental tones. For the $0^{\circ}$ simulation, there is a consistent pattern of increased SPL around both the $3.4 \mathrm{kHz}$ and $4.1 \mathrm{kHz}$ tones found in the experimental results. When looking at its acoustic signal, the high amplitude portions that appear as a result of the 2D wake are dominated by the $4.1 \mathrm{kHz}$ frequency oscillations. The lower amplitude oscillations that occur during the 3D wake are largely dominated by a $2 \mathrm{kHz}$ frequency. The location of the $3.4 \mathrm{kHz}$ tonal behaviour occurs in the transition between the 2D and 3D wake behaviour. The frequency behaviour of the signal is shown in Figure 11. with a $3.4 \mathrm{kHz}$ signal overlaid with the region associated with the 3D to $2 \mathrm{D}$ wake transition, and the $4.1 \mathrm{kHz}$ signal aligned with the acoustic pressure oscillations in the $2 \mathrm{D}$ wake region. This plot serves as a visual representation of the regions associated with the tonal peaks in the narrowband acoustic spectra.

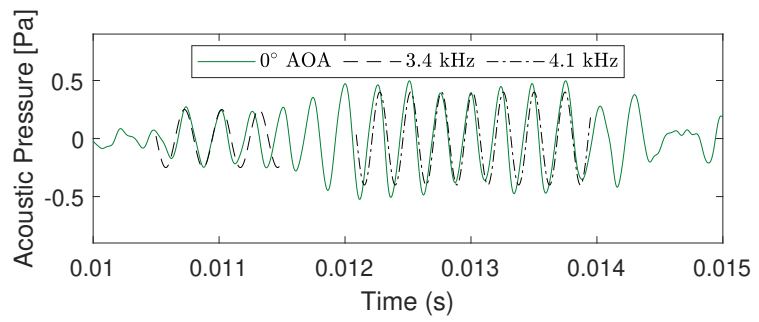

\section{Fig. $110^{\circ}$ AOA location of tonal frequencies in the acoustic signal}

The $1^{\circ}$ acoustic spectra results are shown in Figure $10 \mathrm{~b}$ and compared against the experimental data. The general trend in the data largely agrees with the experimental data. In the higher frequencies, the simulated spectra appears to dip about $10 \mathrm{~dB}$ below the trend in Tam's data. The $1^{\circ} \mathrm{AOA}$ was shown to have the largest tonal noise for the experiments, reaching a peak of $120 \mathrm{~dB}$ for the $3.4 \mathrm{kHz}$ tone. The $1^{\circ} \mathrm{AOA}$ simulations do not predict this tone, which is not surprising when looking at the irregularity of the acoustic signal in comparison with the experimental $1^{\circ}$ signal. The general trend in the simulated data does increase to a maximum value around $3.4 \mathrm{kHz}$, but fails to produce the sharp peak. From the analysis of the $0^{\circ} \mathrm{AOA}$ results, it was proposed that the $3.4 \mathrm{kHz}$ is a result of the transition between the 2D and 3D wake behaviour. The wake analysis in this paper showed that the $1^{\circ}$ AOA simulation spent the majority of time with 3D wake behaviour and cyclically transitioned to partially $2 \mathrm{D}$ behaviour. This would explain why the tone is not produced, since 
it is likely that the strong $3.4 \mathrm{kHz}$ tone found in the experiments is a result of the wake remaining in a transitional state between $2 \mathrm{D}$ and $3 \mathrm{D}$ behaviour.

A common method of comparison of acoustic results is the use of $1 / 3$ octave bands, since it combines the frequency bins from the narrowband spectra in a standard way while also accounting for the increasing frequency ranges for each successive octave. Figure 12a compares the $0^{\circ}$ AOA experimental and simulated results, which shows accurate prediction of the SPL. As mentioned previously, the averaging of multiple signal segments in the experimental data resulted in a much smoother acoustic spectra and well defined tonal peaks. The wider peaks for the simulated data result in an overprediction of the SPL in the tonal regions of the $1 / 3$ octave band data. The trend in the $1 / 3$ octave band for these cases shows an accurate prediction of the acoustic behaviour of the SD 7037 airfoil at $0^{\circ}$. The $1^{\circ} 1 / 3$ octave band comparison is shown in Figure $12 \mathrm{~b}$ and gives a good approximation of the broadband noise produced in the experiments. The inability to simulate the $3.4 \mathrm{kHz}$ tone is shown clearly in this plot, in addition to the peak for the second harmonic of the tone at $6.8 \mathrm{kHz}$, is what causes the large deviation from the experimental results. Again, the tones are not present in the $1^{\circ}$ simulated results because the 3D wake behaviour does not have sufficient periods of time with $2 \mathrm{D}$ behaviour to generate the tonal noise in the acoustic spectra.

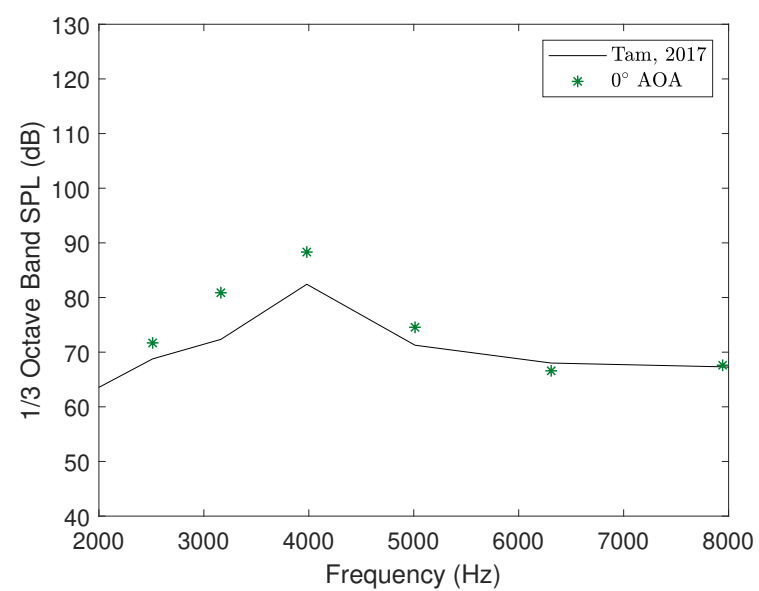

(a)

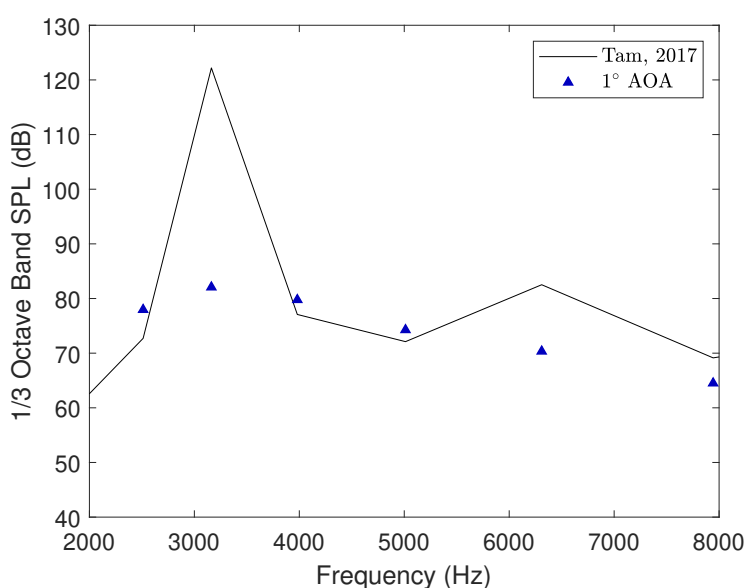

(b)

Fig. $121 / 3$ octave band results for (a) $0^{\circ}$ and (b) $1^{\circ}$ AOA. Results plotted against experimental data of the same angle from Tam [5]

It is clear that this boundary layer and wake behaviour has an impact on the accurate simulation of tonal noise, since the early transition to a 3D wake caused the simulation to produce acoustic results similar to a higher AOA. At a higher AOA the airfoil self noise mechanism switches to TBL-TE, which is only capable of producing broadband noise. Earlier in this paper, the experimental voltage signal for the $3^{\circ}$ AOA exhibited behaviour closest to the simulated acoustic signals for the $1^{\circ}$ results. This also aligns with the $C_{p}$ comparison at the beginning of the results section, where the $1^{\circ}$ simulated results were located between the $1^{\circ}$ and $3^{\circ}$ data sets. The narrowband spectra and the $1 / 3$ octave band results for $1^{\circ} \mathrm{AOA}$ is shown with the experimental $3^{\circ} \mathrm{AOA}$ data in Figure 13 . The $3^{\circ}$ experimental data appears to be a better match for the $1^{\circ}$ AOA simulations, meaning that the flow behaviour for the simulation is that of a higher AOA. Since the $0^{\circ}$ results showed that the $3.4 \mathrm{kHz}$ tone is from the $2 \mathrm{D}$ to $3 \mathrm{D}$ boundary layer and wake transition, and the $1^{\circ}$ results were simulating an AOA closer to $3^{\circ}$, there must be some instability in the simulation that is initiating an early transition to $3 \mathrm{D}$ behaviour. It should also be noted that there is a potential that the closed test section used for the experiments could alter pressure and velocity in the flow and cause a delay in the transition to the 3D turbulent boundary layer behaviour. 


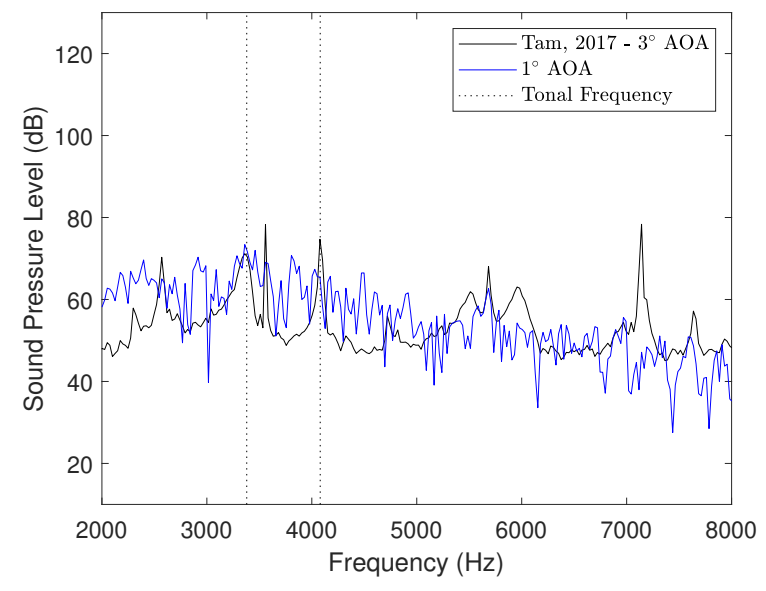

(a) Acoustic Spectra

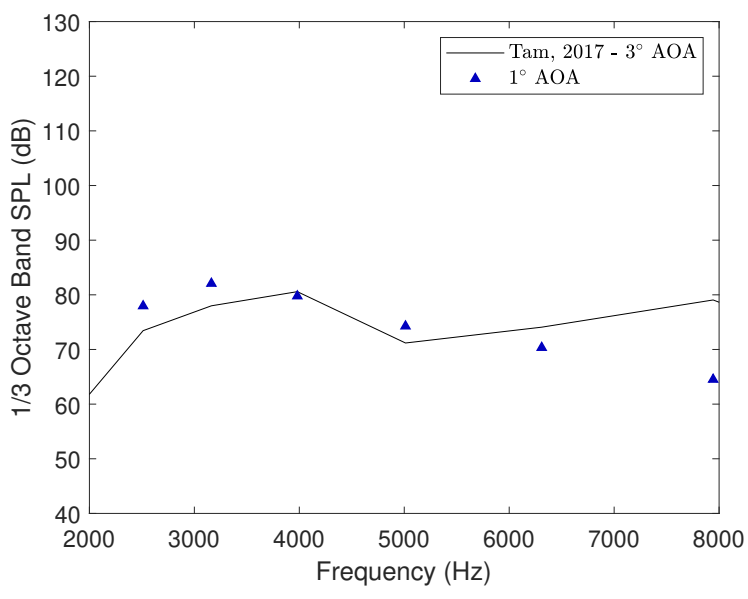

(b) $1 / 3$ Octave Band

Fig. $131^{\circ} \mathrm{AOA}$ acoustic comparison with experimental $3^{\circ} \mathrm{AOA}$ data from Tam [5]

\section{Conclusion}

Overall, the simulation of the SD 7037 airfoil using compressible LES and FW-H produced a good prediction the flow behaviour and corresponding aeroacoustic noise for the given Reynolds number. Unknown sources of instability in the solution caused an alternating 2D and 3D wake behaviour that was reflected in the acoustic results. This instability may be caused by the density changes in the flow due to the density compression and expansion caused by the propagation of acoustic waves or it may be caused by the setup of the simulation itself. Future work is planned to determine the cause of this instability, but for now, it reveals key information about the sources of the tonal noise produced by the LBL-VS self-noise mechanism. The alternating 2D and 3D wake behaviour of the $0^{\circ} \mathrm{AOA}$ simulation showed that the $4.1 \mathrm{kHz}$ tonal noise was generated when $2 \mathrm{D}$ behaviour in the wake passed the TE of the airfoil, and that the $3.4 \mathrm{kHz}$ tonal noise was generated in the transition between the 2D and 3D wake. This is shown clearly in Figure 11 These conclusions drawn from the $0^{\circ}$ simulation explained why the $1^{\circ}$ simulation produced an acoustic spectra similar to the $3^{\circ}$ experimental results. To produce the experimentally measured $1^{\circ}$ tone at $3.4 \mathrm{kHz}$, the boundary layer and wake behaviour would need to stay in a transitional state between the 2D and 3D behaviour, since this was shown to be the source of the tone at that frequency. For the majority of the $1^{\circ}$ simulations, the boundary layer behaviour at the TE and in the wake was 3D, and therefore was not capable of predicting the tonal noise. The boundary layer behaviour for this Re and low AOAs is very sensitive to small changes, so further simulations are required to accurately simulate the flow and acoustics for the sharp tonal noise at $1^{\circ}$ AOA.

Overall, the predictive ability of the LES and FW-H acoustic analogy matches well with experimental flow and acoustic data. Care must be taken when setting up the simulation, since the requirements for the mesh and solution settings are strict. Small differences between the experimental and simulated flow led to missing tonal noise prediction for the $1^{\circ} \mathrm{AOA}$ simulation. Due to the long compute times required for these simulations and the limited time frame for this research, the source of these differences that caused a lack of tonal noise prediction was not determined. Recommendations for future work are to perform a grid independence study along with an analysis to determine the conditions that have the greatest impact on the simulation of the laminar to turbulent boundary layer transition. Future steps required for the application of the LES and FW-H analogy to wind turbine aeroacoustic prediction is to test the model for oscillating AOAs and then application of the model to a rotating blade segment. The results of this research indicates that the power of the aeroacoustic prediction model has the potential to be applied to the prediction of wind turbine noise.

\section{Acknowledgements}

This work was made possible by the facilities of the Shared Hierarchical Academic Research Computing Network (SHARCNET: www.sharcnet.ca) and Compute/Calcul Canada (www.computecanada.ca). Financial support for this 
research was provided by the Natural Science and Engineering Research Council (NSERC), and jointly by the Ontario Graduate Scholarship (OGS) Program and the TD Bank Graduate Scholarship in the Environment.

\section{References}

[1] Selig, M. S., and Giguere, P., "Low Reynolds number airfoils for small horizontal axis wind turbines," Wind Enginering, Vol. 21, No. 6, 1997, pp. 367-380.

[2] ANSYS Academic Research, “ANSYS Fluent Theory Guide, Release 18,” ANSYS Help System, SAS IP Inc., 2018.

[3] Leventhall, G., "Basic Acoustics," Wind Turbine Noise, Multi-Science Publishing Co. Ltd., Essex, 2011, Chap. 1, pp. 1-11.

[4] Bale, A. E., "The Application of MEMS Microphone Arrays to Aeroacoustic Measurements," Ph.D. thesis, University of Waterloo, MASc Thesis, 2011.

[5] Tam, N., “An Aeroacoustic Study of Airfoil Self-Noise for Wind Turbine Applications," Master's thesis, University of Waterloo, 2017.

[6] Ghorbanishohrat, F., "Study of a low Re airfoil considering laminar separation bubbles in static and pitching motion," Ph.D. thesis, University of Waterloo, 2019.

[7] Wagner, S., Bareiss, R., and Guidata, G., Wind Turbine Noise, Springer-Verlag Berlin Heidelberg, Berlin, 1996.

[8] Ffowcs Williams, J., and Hawkings, D., "Sound Generation by Turbulence and Surfaces in Arbitrary Motion," Philisophical Transactions of the Royal Society of London. Series A, Mathematical and Physical Sciences, Vol. 264, No. 1151, 1969, pp. 321-342.

[9] Farassat, F., "Derivation of Formulations 1 and 1A of Farassat," Nasa/TM-2007-214853, Vol. 214853, No. March, 2007, pp. $1-25$.

[10] Casey, M., and Wintergertse, T., "Quality and Trust in Industrial CFD" Best Practice Guidelines," Tech. rep., European Research Community on Flow, Turbulence and Combustion (ERCOFTAC), 2000.

[11] SHARCNET, "SHARCNET, Ontario's network of high-performance computer clusters," , 2018. URL www . sharcnet . ca

[12] ComputeCanada, "Compute Canada, Advanced computing services in support of research,", 2018. URL www . computecanada. ca

[13] Selig, M. S., Lyon, C. A., Giguere, P., Ninham, C. P., and Guglielmo, J., Summary of low-speed airfoil data, Vol. 2, SoarTech Publications, VA, 1996.

[14] Gharali, K., "Pitching airfoil study and freestream effects for wind turbine applications," Ph.D. thesis, University of Waterloo, 2013. 